\title{
Slitless spectroscopy with the James Webb Space Telescope Near-Infrared Camera (JWST NIRCam)
}

\author{
Thomas P. Greene ${ }^{\mathrm{a}}$, Laurie Chu ${ }^{\mathrm{b}}$, Eiichi Egami ${ }^{\mathrm{c}}$, Klaus W. Hodapp ${ }^{\mathrm{b}}$, Douglas M. Kelly ${ }^{\mathrm{c}}$, \\ Jarron Leisenring ${ }^{\mathrm{c}}$, Marcia Rieke ${ }^{\mathrm{c}}$, Massimo Robberto ${ }^{\mathrm{d}}$, Everett Schlawin ${ }^{\mathrm{c}}$, and John \\ Stansberry ${ }^{\mathrm{d}}$ \\ ${ }^{a}$ NASA Ames Research Center, Moffett Field, CA, USA \\ bInstitute for Astronomy, University of Hawai'i, Honolulu, HI, USA \\ 'Steward Observatory, University of Arizona, Tucson, AZ, USA \\ dSpace Telescope Science Institute, Baltimore, MD, USA \\ July 19, 2016 draft for SPIE Astronomical Telescopes + Instrumentation 2016
}

\begin{abstract}
The James Webb Space Telescope near-infrared camera (JWST NIRCam) has two 2'.2 $\times 2.2$ fields of view that are capable of either imaging or spectroscopic observations. Either of two $R \sim 1500$ grisms with orthogonal dispersion directions can be used for slitless spectroscopy over $\lambda=2.4-5.0 \mu \mathrm{m}$ in each module, and shorter wavelength observations of the same fields can be obtained simultaneously. We present the latest predicted grism sensitivities, saturation limits, resolving power, and wavelength coverage values based on component measurements, instrument tests, and end-to-end modeling. Short wavelength $(0.6-2.3 \mu \mathrm{m})$ imaging observations of the $2.4-5.0 \mu \mathrm{m}$ spectroscopic field can be performed in one of several different filter bands, either in-focus or defocused via weak lenses internal to NIRCam. Alternatively, the possibility of $1.0-2.0 \mu \mathrm{m}$ spectroscopy (simultaneously with $2.4-5.0 \mu \mathrm{m}$ ) using dispersed Hartmann sensors (DHSs) is being explored. The grisms, weak lenses, and DHS elements were included in NIRCam primarily for wavefront sensing purposes, but all have significant science applications. Operational considerations including subarray sizes, and data volume limits are also discussed. Finally, we describe spectral simulation tools and illustrate potential scientific uses of the grisms by presenting simulated observations of deep extragalactic fields, galactic dark clouds, and transiting exoplanets.
\end{abstract}

Keywords: James Webb Space Telescope, JWST, NIRCam, grisms, slitless spectroscopy

\section{INTRODUCTION}

The near-infrared camera (NIRCam) of the James Webb Space Telescope (JWST) has numerous flexible modes that provide wide-field imaging through narrow, medium or wide filters, coronagraphic imaging, and slitless spectroscopy. ${ }^{1-4}$ These and other previous works have described its wide-field and coronagraphic imaging capabilities well, but little practical information has been published on using NIRCam in its spectroscopic modes. Long-wave $(\mathrm{LW} ; \lambda=2.4-5.0 \mu \mathrm{m}) \mathrm{Si}_{\text {grisms }}{ }^{5}$ and short-wave (SW; $\lambda=1-2 \mu \mathrm{m}$ ) Dispersed Hartmann Sensors (DHSs) were developed for the purposes of wavefront sensing and telescope primary segment phasing with NIRCam. ${ }^{6}$ The LW grisms have been approved and are being supported for scientific use. The SW DHSs are not yet supported but are being considered for scientific use during Cycle 1 and later observations.

Both of these optics give NIRCam the ability to perform slitless spectroscopic observations. Full-field slitless observations produce spectral images that contain a spectrum for nearly all objects in the NIRCam imaging field. This is more complete spatially that using a spectrometer with apertures (e.g., opening the MSA masks in the JWST NIRSpec instrument) and is more suitable for mapping regions of the sky (e.g., to search for high redshift emission line objects). When implemented in a camera like NIRCam, the spectral bandpass of slitless exposures can be selected with filters. This can be used to limit the content (e.g., spectral features or redshift range) as well as optimize the sensitivity and extent of spectra. Overlapping spectra of multiple objects can often be resolved

Send correspondence to T.P.G.: E-mail: tom.greene@nasa.gov, Telephone: +0016505395244 
by taking separate slitless spectral exposures with orthogonal dispersion directions. Slitless spectroscopy is also valuable for high precision observations of bright stars (e.g., time series spectra of transiting planets) because there is no slit to impart artifacts into the spectrum in the presence of telescope jitter or if there are variations in the PSF with wavelength (e.g., diffraction-limited images). Slitless spectra do see the full background of their undispersed bandpasses and therefore have lower sensitivities than those of slit spectrographs. However, the low background of JWST allows NIRCam slitless spectra to have sensitivities within a factor of a few of NIRSpec in the $\lambda=2.4-5.0 \mu \mathrm{m}$ range.

The NIRCam instrument is composed of two nearly identical optical modules (A and B), each having a 2 ' $2 \times 2$ '2 field of view (FOV). Wavelengths $\lambda<2.4 \mu \mathrm{m}$ are imaged by the short-wavelength (SW) channel of each module which has a focal plane of four butted $2048 \times 2048(2040 \times 2040$ active $)$ pixel HAWAII-2RG detectors with a plate scale of 32 mas pixel $^{-1}$. A dichroic beamsplitter sends longer wavelengths to the long-wavelength $(\mathrm{LW})$ channel that has a single $2048 \times 2048(2040 \times 2040$ active $)$ pixel HAWAII-2RG detector focal plane with a plate scale of 65 mas pixel ${ }^{-1} .^{1,3}$ This allow simultaneous SW and LW imaging of the same NIRCam field with each module. The pupil wheels in the LW channels of each module are equipped with two identical Si grisms with perpendicular orientations so that light is dispersed along either the rows ( $\mathrm{R}$ grisms) of columns (C grisms) of their detectors. Each module also has two SW pupil wheel positions containing a DHS with 10 apertures, with each aperture containing a grism and a wedge to spatially separate the 10 spectra. The dispersions of all 10 DHS grisms in each wheel position are oriented either along rows (DHS0) or else rotated $60^{\circ}$ (DHS60). ${ }^{6}$ Each grism in each DHS is mapped to a pair of telescope segment edges and in aggregate subtend approximately $40 \%$ of the geometric telescope pupil area.

We present practical information on using NIRCam's spectroscopic modes in the following sections. First we present basic spectroscopic capabilities and performance limits of the grisms and DHS elements. Next we discuss operational considerations including using these elements simultaneously. We conclude with simulations of wide-field multi-object spectra of deep extragalactic fields and galactic dark clouds as well as single-object simulations of transiting exoplanet spectra.

\section{SPECTROSCOPIC CAPABILITIES}

\subsection{Basic Performance Parameters}

The LW grisms were designed to have undeviated wavelengths of $4.0 \mu \mathrm{m}$ and to provide a dispersion of 10.0 $\AA$ pixel $^{-1}$ in NIRCam. ${ }^{4}$ Dispersions for all four grisms (two in each module) were measured to be within $1 \%$ of this value during JWST science instrument testing at NASA Goddard Space Flight Center (GSFC) during the summer of 2014.

Filters are used in series with grisms to select the desired spectral range of the observation. The NIRCam LW filter wheel contains filters that are medium $(\lambda / \Delta \lambda \sim 10-20)$, wide $(\lambda / \Delta \lambda \sim 4)$, and double-wide $(\lambda / \Delta \lambda \sim 2)$ bandpasses*, and any of these can be used with the grisms in principle. A subset of these is being defined for science applications. All LW narrow-band filters reside in the same pupil wheel as the grisms, so these cannot be used in series with the grisms. Each grism spans the entire telescope pupil, and any source in the NIRCam imaging field will have its light dispersed by a grism that is selected in the pupil wheel. Some of the dispersed spectrum may land outside of the FOV and not be recorded by the detector depending on the object field position and the selected filter (See Section 3). The image and extracted spectrum of a NIRCam Module A LW grism exposure taken during the JWST instrument test campaign at NASA GSFC in 2014 is shown in Figure 1. This figure also shows the layout of an object's LW R grism spectra spectrum relative to its direct image position for different filters.

The SW DHS grisms have undeviated wavelengths of $1.36 \mu \mathrm{m}$, and their dispersions have been measured to be $2.90 \AA$ (Module A) and $2.91 \AA$ (Module B) per NIRCam SW pixel. Therefore two NIRCam SW filters plus their $\sim 5^{\prime \prime}$ gap span approximately $1.235 \mu \mathrm{m}$ in wavelength. DHS elements will be typically used with F150W2 filters which have bandpasses from 1.01 to $2.33 \mu \mathrm{m}$, so $\lambda=1-2 \mu \mathrm{m}$ DHS spectra can be imaged simultaneously on 2 adjacent SW detectors. Observing an object with the DHS requires that it be located in a specific region

\footnotetext{
*see http://www.stsci.edu/jwst/instruments/nircam/instrumentdesign/filters/
} 

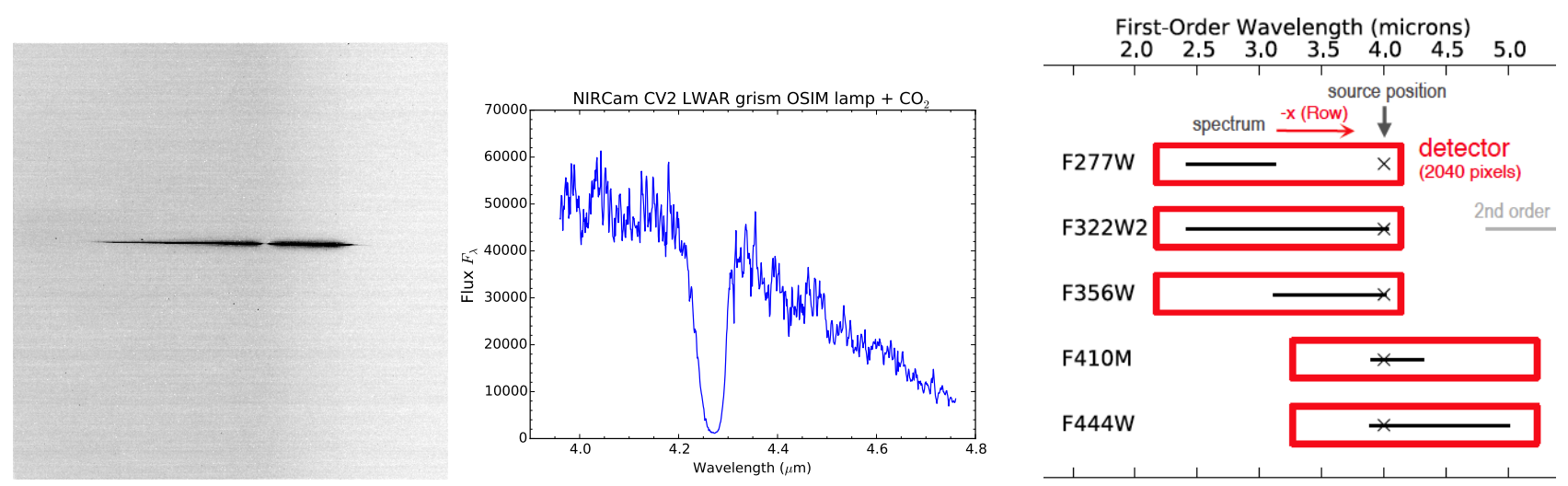

Figure 1. Left: NIRCam spectral image of the OSIM super-continuum lamp taken with the LWA R grism and F444W filter during JWST instrument instrument testing at NASA GSFC in August 2014. Wavelength increases to the left (-X direction). Center: Extracted spectrum from the image with an approximate wavelength calibration applied. The continuum decreases toward longer wavelengths due to low fiber transmittance, and the broad feature near $4.27 \mu \mathrm{m}$ is due to $\mathrm{CO}_{2}$ absorption. Both of these features are artifacts of the test equipment and not NIRCam itself. Right: Layout of an object's LW R grism spectra spectrum relative to its direct image position for different filters (image courtesy of D. Coe).

of the NIRCam field to ensure that all 10 DHS spectra are imaged onto the SW detectors over their entire $\lambda=1-2 \mu \mathrm{m}$ regions. Multiple objects in the field can also produce overlapping DHS spectra. Therefore DHS observations are best suited for single-object spectroscopy, and relatively bright stars are required to achieve high signal-to-noise. Figure 2 shows the layout of the DHS0 elements on the JWST pupil and an image of the resultant 10 DHS spectra.

Both the LW grisms and DHSs are usually operated in first order $(m=1)$ where they have maximum efficiency. Their high dispersions, the finite size of the NIRCam field and detectors, and the limited bandpasses of the series filters all limit the detection of other orders. It is only possible to detect $m \neq 1$ light when using the F322W2 filter with an LW grism. In that case, an object at the right location can produce a spectrum that is $\mathrm{m}=1$ at long wavelengths and $\mathrm{m}=2$ at short wavelengths (relative wavelengths within the F322W2 bandpass; see Figure 1 right panel). The broad bandpass of the F150W2 filter will cause DHS $\lambda<2 \mu \mathrm{m}, \mathrm{m}=2$ spectra to overlap partially with $\lambda>2 \mu \mathrm{m}, \mathrm{m}=1$ DHS spectra. In particular, $\mathrm{m}=1,2.02-2.33 \mu \mathrm{m}$ wavelengths will be contaminated with $\mathrm{m}=2$ light from $1.01-1.165 \mu \mathrm{m}$ wavelengths.

\subsection{Performance: Resolution, Sensitivities and Saturation Limits}

The transmission and reflectance values of all NIRCam optical components used for imaging observations have been tested, and the composite throughput values for Module A (including the OTE reflectivity as well as NIRCam detector quantum efficiency) are shown in Figure 3. This figure also shows the theoretical first-order Module A LW grism efficiency, which was validated with measurements at two wavelengths near $3 \mu \mathrm{m}$ during pre-delivery instrument testing at the Lockheed Martin Advanced Technology Center. The grism efficiency must be multiplied by the NIRCam + OTE curve for the chosen series filter to produce the system throughput at each wavelength. Module A LW grisms are anti-reflection coated on both sides, while those in the B module are coated only on their flat (non-grooved) sides. Therefore the Module B LW grism efficiencies are $\sim 0.74$ times that of the Module A ones, and the Module B ones produce some ghosts of bright spectra.

All NIRCam spectroscopy is conducted without using slits or other focal plane apertures, so the spectral resolving power $R$ of its grism or DHS observations are determined by the dispersion, wavelength, its circular pupil, ${ }^{7}$ detector sampling, and object size. The basic equation for point-source resolving power $R \equiv \lambda / \Delta \lambda$ dictates that $R$ increases with wavelength for a constant spectral bandpass $\Delta \lambda$ (e.g., an undersampled 2 pixel PSF), but this is flattened by diffraction for the LW grisms at wavelengths $\lambda \gtrsim 4 \mu \mathrm{m}$ where the well-sampled NIRCam PSF causes point sources to subtend more pixels. The resultant spectroscopic resolving power for LW 

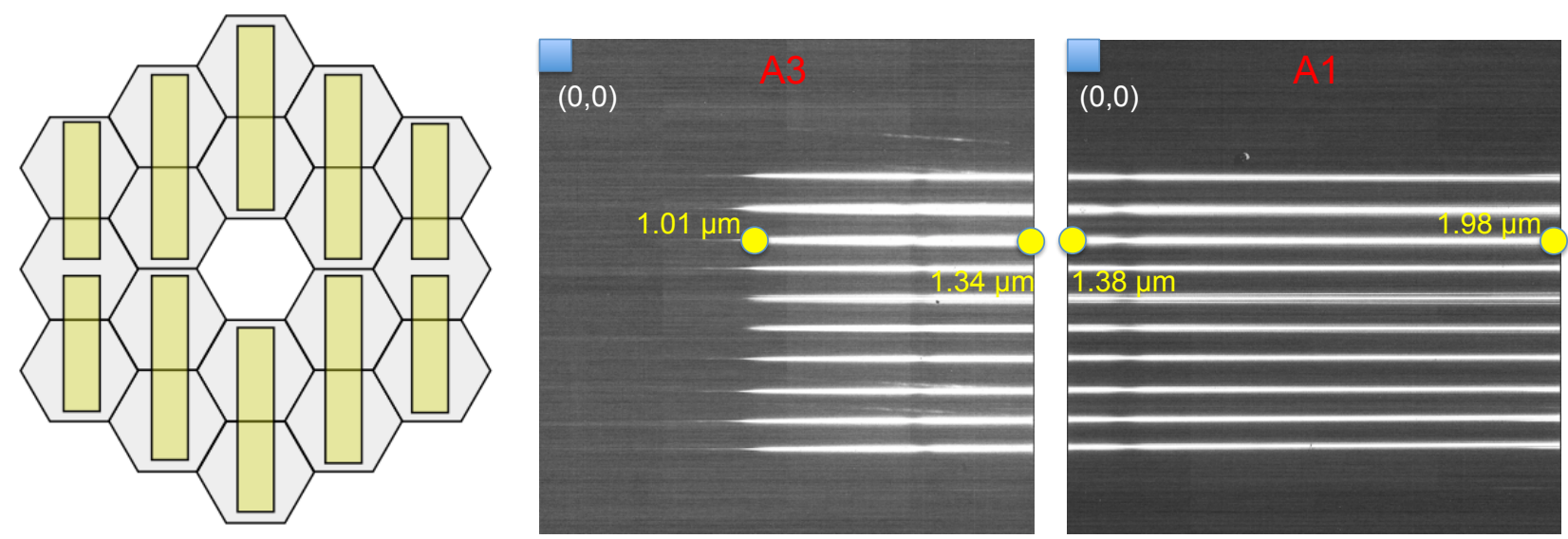

Figure 2. Left: Schematic projection of the SW DHS0 elements onto the JWST primary mirror segments. Each of the 10 DHS elements span 2 segment edges, and approximately $40 \%$ of the total optical telescope element (OTE) aperture is seen by all DHS elements in total. Obscurations by the secondary supports are not shown. A NIRCam image of all 10 DHS0 $\lambda=1-2 \mu \mathrm{m}$ spectra is also shown, with the shorter wavelength A3 detector image in the center panel and the longer wavelength A1 detector image in the right panel. Wavelength increases from left to right in both detector images. The source was positioned such that the $1.01 \mu \mathrm{m}$ short half-power wavelength of the series F150W2 filter appears near the center of the A3 detector (center panel), and its $2.33 \mu \mathrm{m}$ long half-power wavelength falls beyond the right edge of the A1 detector (right panel). The gap between the 2 detectors is approximately 150 pixels $\left(\sim 5^{\prime \prime}\right.$ on the sky), corresponding to about $0.045 \mu \mathrm{m}(45 \mathrm{~nm})$ in wavelength.
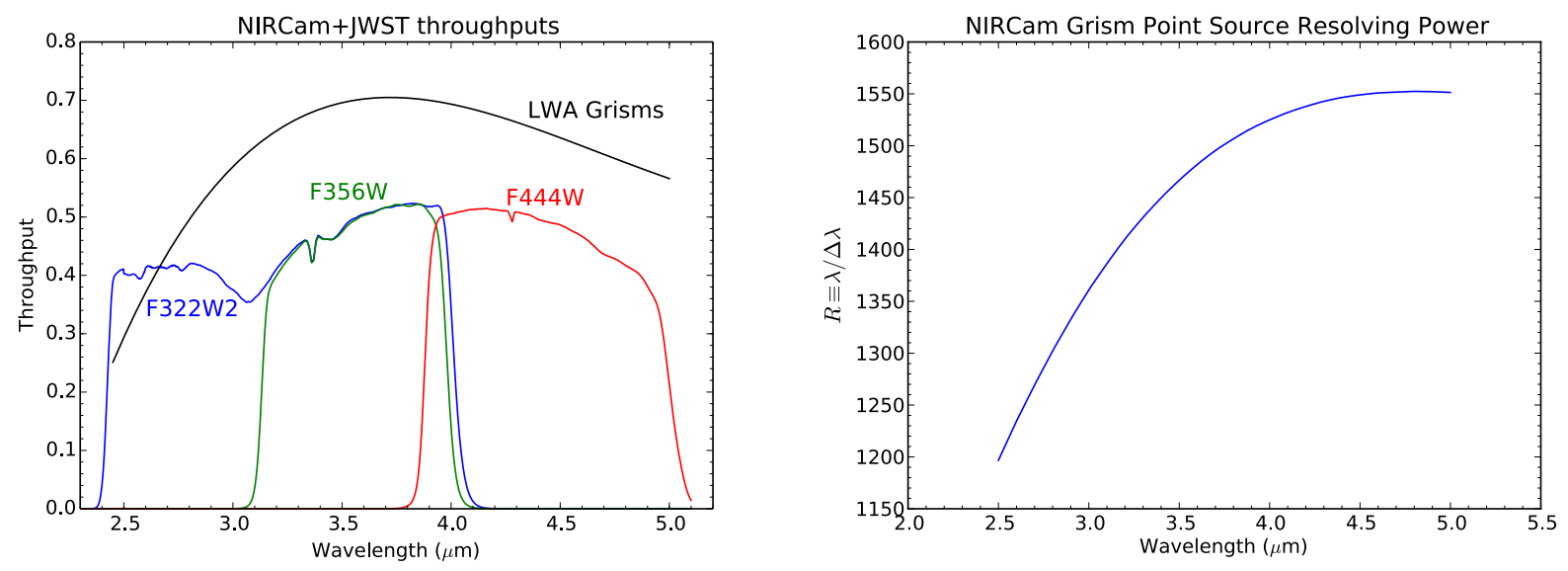

Figure 3. Left: Total system throughput including all OTE and NIRCam optics and the detector quantum efficiency for several NIRCam filters. The theoretical LW grism efficiency curve (shown for the A module) must be multiplied by the filter curves to produce the system throughput at each wavelength. The Module B LW grisms are anti-reflection coated on only 1 side and therefore have throughputs approximately 25\% lower than the LWA grisms. Right: Grism FWHM spectral resolving power vs. wavelength for point sources, limited by pixel sampling of the PSF at shorter wavelengths $(\lambda \lesssim 4 \mu \mathrm{m})$ and limited by the circular beam factor ${ }^{7}$ and diffraction at longer wavelengths $(\lambda \gtrsim 4 \mu \mathrm{m})$.

grism observations of point sources is shown in the right panel of Figure 3. The pixel-limited resolving power (strongest at $\lambda \lesssim 4 \mu \mathrm{m}$ ) may be improved with dithering of multiple observations.

Table 1 gives the $10 \sigma$ point-source continuum sensitivities for the Module A grisms using a 2 (spectral) $\times 5$ (spatial) pixel extraction aperture in 10,000 s of integration time. Unresolved point source emission line sensitivities are also given, using the spectral resolving power computed for each wavelength (right panel of Figure 3). All sensitivities were calculated for medium zodiacal background levels using either the F322W2 or F444W filter in series, as identified in the table. These sensitivities are a factor of $\sim 5$ worse than expected for 
Table 1. Module A Grism performance table ${ }^{\mathrm{a}}$

\begin{tabular}{|r|r|r|r|r|l|}
\hline$\lambda(\mu \mathrm{m})$ & $\mathrm{F}_{\text {cont }}\left(\mu \mathrm{Jy}^{\mathrm{b}}\right.$ & $\mathrm{F}_{\text {line }}\left(\mathrm{W} \mathrm{m}^{-2}\right)^{\mathrm{c}}$ & $K_{\text {sat }}(\mathrm{A} 0 \mathrm{~V})^{\mathrm{d}}$ & $K_{\text {sat }}(\mathrm{M} 2 \mathrm{~V})^{\mathrm{d}}$ & Filter $^{\mathrm{e}}$ \\
\hline 2.5 & 11.1 & $1.09 \mathrm{E}-20$ & 4.3 & 4.3 & F322W2 \\
\hline 2.7 & 8.7 & $7.35 \mathrm{E}-21$ & 4.5 & 4.6 & F322W2 \\
\hline 2.9 & 8.0 & $5.98 \mathrm{E}-21$ & 4.3 & 4.5 & F322W2 \\
\hline 3.1 & 7.9 & $5.22 \mathrm{E}-21$ & 4.2 & 4.4 & F322W2 \\
\hline 3.3 & 6.7 & $3.97 \mathrm{E}-21$ & 4.2 & 4.5 & F322W2 \\
\hline 3.5 & 6.5 & $3.45 \mathrm{E}-21$ & 4.0 & 4.3 & F322W2 \\
\hline 3.7 & 6.3 & $3.05 \mathrm{E}-21$ & 3.9 & 4.2 & F322W2 \\
\hline 3.9 & 7.0 & $3.11 \mathrm{E}-21$ & 3.6 & 3.9 & F322W2 \\
\hline 4.1 & 12.1 & $4.99 \mathrm{E}-21$ & 3.5 & 3.8 & F444W \\
\hline 4.3 & 13.5 & $5.18 \mathrm{E}-21$ & 3.2 & 3.5 & F444W \\
\hline 4.5 & 15.1 & $5.38 \mathrm{E}-21$ & 2.9 & 3.0 & F444W \\
\hline 4.7 & 19.1 & $6.38 \mathrm{E}-21$ & 2.5 & 2.7 & F444W \\
\hline 4.9 & 25.1 & $7.88 \mathrm{E}-21$ & 2.2 & 2.3 & F444W \\
\hline
\end{tabular}

${ }^{a}$ Module B grisms will have sensitivities approximately 1.16 times higher (worse) and saturation limits 0.33 mag brighter.

b $10 \sigma$ point-source continuum sensitivities for $10,000 \mathrm{~s}$ integrations using a 2 (spectral) $\times 5$ (spatial) pixel extraction aperture.

c $10 \sigma$ point-source unresolved emission line sensitivities for 10,000 s integrations using the actual spectral resolving power at this wavelength (right panel of Figure 3).

${ }^{\mathrm{d}}$ K-band magnitudes for saturation ( $80 \%$ full well or 65,000 electrons) for $0.68 \mathrm{~s}$ integrations ( 2 reads) of $2048 \times 64$ pixel regions in stripe mode ( 4 outputs). Larger subarrays or use of only 1 output will result in fainter bright limits.

e Narrower filters will have similar saturation values and somewhat lower (better) sensitivities.

the JWST NIRSpec instrument ${ }^{\dagger}$, but NIRSpec cannot be operated in a true slitless mode. Lower background conditions than those assumed here will give sensitivities that are up to a factor of $\sim 1.4$ lower (better), and higher background conditions will result in sensitivities that are up to a factor of $\sim 1.4$ higher (worse). Using narrower band filters (i.e., F356W instead of F322W2, or F430M instead of F444W) can improve sensitivities by a factor of $\sim 1.2-2$. Table 1 also gives saturation values for $0.68 \mathrm{~s}$ integrations, 2 frame times for $2048 \times$ 64 pixel subarrays with the detector operated with four outputs (stripe mode). Larger subarray stripes $(2048 \times$ $>64$ pixels) will result in fainter bright limits, and operating in window mode (using a single detector output) will make the bright limits 1.5 mag fainter also. The Module B grisms will have sensitivities approximately 1.16 times higher (worse) and saturation limits 0.33 mag brighter than the ones tabulated for Module A.

As seen in Figure 2, the DHS elements subtend about $40 \%$ of the OTE pupil, and they have transmission values on the order of 0.6. This and the fact that each DHS component produces a separate spectrum results in relatively low photon fluxes in each detector pixel. Consequently, full-array DHS exposures with 2 frame integrations (21.4 s integration times) have a saturation limit ( $80 \%$ full well) of $5.0-3.25$ mag (Vega) for a G2V star over the $1.0-2.0 \mu \mathrm{m}$ wavelength range, respectively. This improves to $1.2--0.3$ mag when only a $2048 \times$ 64 pixel detector region is operated in stripe mode (four outputs), but this configuration will record only 1 or 2 of the 10 DHS spectra.

\section{SPECTROSCOPIC OPERATIONS}

The NIRCam LW grisms are expected to be used in either of two modes: single-object time-series slitless spectroscopy or wide-field (multi-object) slitless spectroscopy. The layout of the grism dispersions on the NIRCam LW detectors and in the JWST focal plane is shown in Figure 4. A single object can be positioned at the

\footnotetext{
${ }^{\dagger}$ see http://www.stsci.edu/jwst/instruments/nirspec/sensitivity/
} 

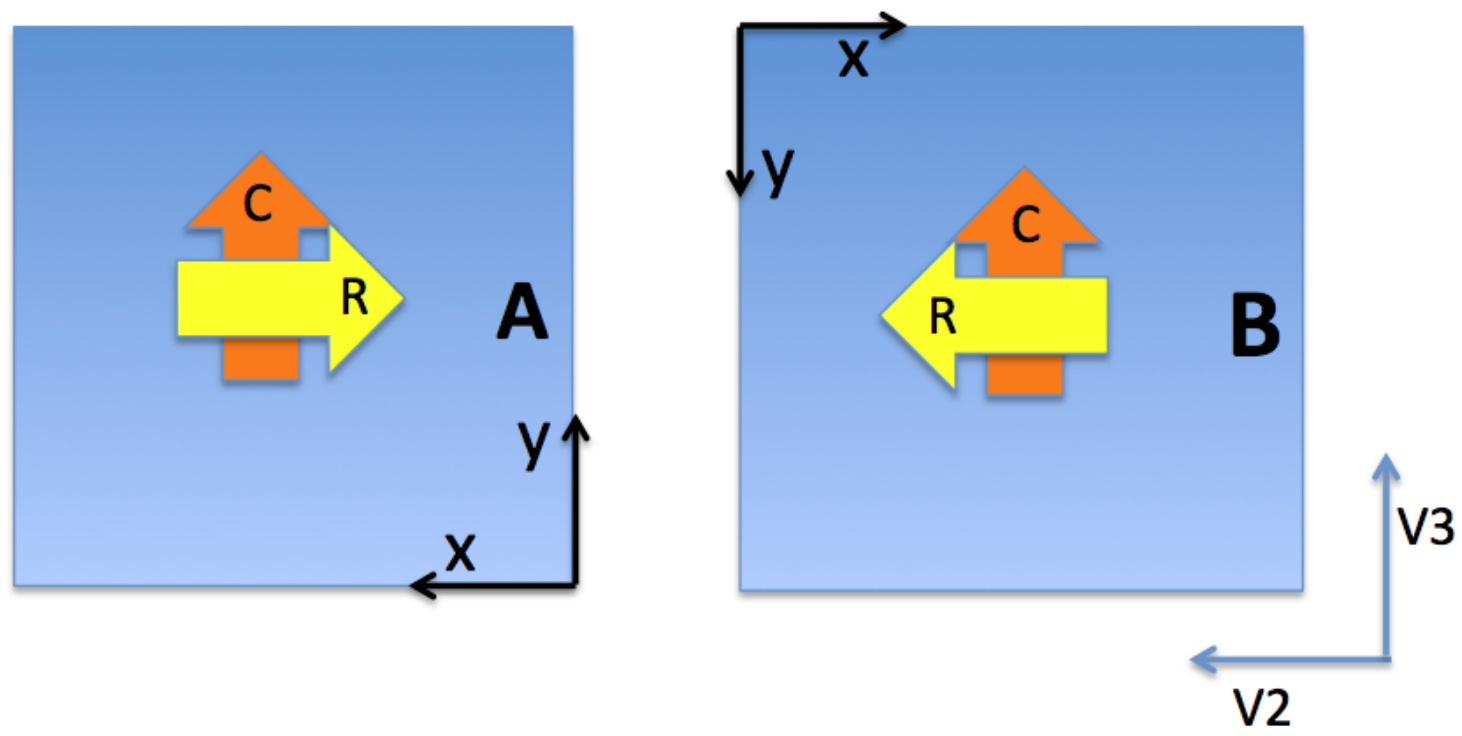

Figure 4. Grism dispersion orientations along detector axes (x, y) and the JWST focal plane (V2, V3). The arrows point in the direction of increasing wavelength for $\mathrm{R}$ and $\mathrm{C}$ grisms in modules $\mathrm{A}$ (right) and $\mathrm{B}$ (left). Each module has a 2 '. $2 \times 2$ '. 2 imaging field of view.

Table 2. Filters available for use with LW grisms in Cycle 1

\begin{tabular}{|l|r|r|r|r|l||}
\hline Filter Name $^{\mathrm{a}}$ & $\lambda_{1}(\mu \mathrm{m})^{\mathrm{b}}$ & $\lambda_{2}(\mu \mathrm{m})^{\mathrm{c}}$ & \# dispersed pixels & \# pixels $/ 2048^{\mathrm{d}}$ & Mode $^{\mathrm{e}}$ \\
\hline F277W & 2.416 & 3.127 & 711 & 0.35 & $\mathrm{TS}+\mathrm{WF}$ \\
\hline F322W2 & 2.430 & 4.013 & 1583 & 0.77 & $\mathrm{TS}+\mathrm{WF}$ \\
\hline F356W & 3.140 & 3.980 & 840 & 0.41 & $\mathrm{TS}+\mathrm{WF}$ \\
\hline F444W & 3.880 & 4.986 & 1106 & 0.54 & $\mathrm{TS}+\mathrm{WF}$ \\
\hline F430M & 4.167 & 4.398 & 231 & 0.11 & $\mathrm{WF}$ \\
\hline F460M & 4.515 & 4.747 & 232 & 0.11 & $\mathrm{WF}$ \\
\hline
\end{tabular}

${ }^{\text {a }}$ All LW M filters will also likely be available in wide-field mode; F430M and F460M are expected to be popular and are included for illustrative purposes

${ }^{\mathrm{b}}$ Half-power wavelength (blue side)

${ }^{\mathrm{c}}$ Half-power wavelength (red side)

${ }^{\mathrm{d}}$ Fraction of the detector that a continuum spectrum occupies in the dispersion direction

e $\mathrm{TS}=$ single-object time series and $\mathrm{WF}=$ wide field modes

appropriate point to have its entire spectrum (as limited by the chosen filter) fall onto the detector array. The grisms can be operated in series with any of the filters in the NIRCam LW pupil wheel in principle, but several of these would be redundant. Table 2 lists all wide and some of the medium-band the filters that will likely be enabled for use with the grisms during JWST observing Cycle 1. We expect those to be most popular, and the remaining medium-band LW filters are also expected to be available for use in wide-field mode in Cycle 1.

Spectra of multiple objects in the imaging field will be recorded, and large sky areas can be mapped with multiple telescope pointings. The DHS elements require that a single object be positioned near a single field point to collect its entire $\lambda=1-2 \mu \mathrm{m}$ spectrum from all 10 DHS elements. Multiple objects in the field can easily produce overlapping DHS spectra, so DHS observations should be restricted to single objects, compact regions, or relatively uncrowded fields (if and when the DHSs are supported for scientific use).

The NIRCam detectors will be operated in their normal MUTIACCUM modes ${ }^{8}$ during spectroscopic observations. High-precision time-series grism observations of bright objects (e.g., host stars of transiting planets) 
will likely require using a limited detector region to prevent saturation. Sub-regions consisting of $64,128,256$, or the full 2048 detector rows will be supported for single-object time-series LW grism observations. These can be read out either using all 4 outputs (stripe mode; spanning all 2048 columns) or else in subarray (or window) mode using a single output and possibly a smaller number of columns. Using the combination of stripe mode and 64 detector rows gives the fastest readout time and provides the best bright limits (appearing in Table 1). Subarrays with a smaller dimension of 64 pixels are also likely to be supported in wide-field mode, and we expect that these will be used primarily for calibration purposes.

LW grism spectral observations can be partnered with either simultaneous DHS spectra (if and when DHS use becomes supported) or imaging observations of the same field in the SW channels. SW imaging observations can be conducted in-focus using only filters (in both LW spectroscopic modes), or else out of focus using a weak lens with a filter in series (single-object time-series mode only). Given the flux differences between dispersed spectra and in-focus images, we expect that many single-object point source observers will opt for using a weak lens in series with a filter in the SW channel for simultaneous observations with the LW grisms.

All NIRCam detectors will be operated with the same readout pattern and subarray size, so SW observations will use the same size detector regions as used for the LW grisms. This will limit the number of DHS spectra recorded unless the full frame readout mode is selected. When collecting simultaneous SW weak lens (8 waves of defocus at $2.12 \mu \mathrm{m}$ ) observations, the smallest supported subarray size will be 160 pixels. This is large enough to capture $98 \%$ of the light from the source, while providing adequate area around the PSF for background measurements $(190 \times 190$ pixel regions collect nearly all light, requiring use of a larger subarray). As noted earlier, the subarrays used in the SW and LW channels will have the same dimensions. However, their locations on the LW and SW detectors can be individually adjusted. This is of particular importance if simultaneous observations using the DHSs and LW grisms become a supported mode; it allows the location of the SW subarrays to be tailored to include the best centered and brightest DHS spectra while keeping the LW grism spectrum centered in the LW subarray. When SW data are taken in parallel with the LW grism data in any mode, particular care must be exercised to ensure that the single set of exposure parameters provide adequate dynamic range in both the SW and LW channels.

Short single-object time-series integrations will likely use the RAPID or BRIGHT1 multiaccum modes that save every or every other frame read. This will produce a large amount of data, especially when conducting simultaneous DHS and LW grism observations since they will use three detector arrays (if DHS use is implemented). The on-board solid state data recorder can store $7-10$ hours of RAPID and for 3 detector arrays operated in stripe mode (four outputs), and $28-40$ hours when operated with a single detector output. JWST is expected to download data every 12 hours, so all but the longest transits of the brightest stars can be observed with both the LW grisms and SW DHSs.

\section{SIMULATIONS OF SPECTROSCOPIC OBSERVATIONS}

We have developed software tools to simulate NIRCam spectroscopic observations in order to assess expected performance and to plan using NIRCam in both single-object and multi-object wide-field slitless spectroscopy modes. We describe these tools and present some results in this section.

The first simulator is designed to produce spectral images from multiple objects distributed in 2 spatial dimensions within a given NIRCam field. We have implemented this using the aXeSIM package, first developed for simulating HST WFC3 slitless spectroscopic images ${ }^{\ddagger}$. Spectra of individual objects can be extracted from simulated images using conventional astronomical data tools. We have produced aXeSIM configuration files that represent the expected signal and noise performance of the NIRCam LW grisms as we expect them to be used on JWST. In addition to producing the wide-field slitless spectroscopy simulations in this section, we have used aXeSIM to produce the NIRCam performance estimates given in Table 1. These estimates agree with those of SimNRC, the internal NIRCam team exposure time calculator, to within $\sim 10 \%$. Please contact T. Greene if you would like a package of the NIRCam LWA R grism configuration files for use with aXeSIM (contact information on first page of manuscript).

\footnotetext{
${ }^{\ddagger}$ see http://axe.stsci.edu/axesim/
} 
We have also developed a second simulator that is used to estimate the signals and noise expected for high precision time series observations of transiting planet spectra. These codes include the same throughputs (e.g., Figure 3) used in the aXeSIM configuration files, and they also include an expected systematic noise floor in addition to the regular noise terms for detector read noise, dark noise, and observatory background noise components. One-dimensional simulations of exoplanet transmission (in transit) or emission (in secondary eclipse) spectra are produced, and they are described further in a recent scientific publication. ${ }^{9}$

\subsection{Wide-Field Slitless Spectroscopy of Extragalactic Fields}

$J W S T$ is expected to revolutionize the study of high-redshift galaxies, allowing us to spectroscopically study familiar rest-frame optical emission lines at high redshifts. With a wavelength coverage of $2.4-5 \mu \mathrm{m}$, the NIRCam LW grism mode can detect $\mathrm{H} \alpha$ at $z=2.7-6.6$, [O III] $5007 \AA$ at $z=3.8-9.0$, and [O II] $3727 \AA$ at $z=5.4-12.4$. Although NIRSpec will be the preferred choice for most extragalactic spectroscopic observations because of its superior sensitivity and wider wavelength coverage $(1-5 \mu \mathrm{m}$ for $R=1000 / 2700 ; 0.7-5.0 \mu \mathrm{m}$ for $R=100$ ), the NIRCam LW grism mode does offer some interesting and potentially powerful capabilities. For example, the NIRCam LW grism mode will allow: (1) blind searches for strong line emitters at high redshift, (2) spatially-resolved spectroscopy for a large number of galaxies simultaneously, and (3) spectroscopic surveys without pre-imaging data (well suited for parallel programs).

Figure 5 shows the simulation of a 2-hour NIRCam LW grism exposure taken over the eXtreme Deep Field ${ }^{10}$ (XDF) using the F356W filter. Here, the redshifts of all the sources have been set to 6 artificially to illustrate the detectability of $\mathrm{H} \beta$ and [O III] 4959/5007 $\AA$ lines as function of source brightness. In this image, the continuum is visible down to $\sim 24 \mathrm{AB}$ mag $(\sim 1 \mu \mathrm{Jy})$ while emission lines are visible down to $\sim 2 \times 10^{-18} \mathrm{erg} \mathrm{cm}^{-2} \mathrm{~s}^{-1}$. Although the confirmation of actual sensitivities has to wait for in-orbit observations, this simulation clearly demonstrates that NIRCam LW grism observations of extragalactic fields will produce rich data sets.

Recent studies indicate that the rest-frame optical emission lines of some $z \sim 7$ galaxies are quite large. ${ }^{11}$ The combined rest-frame equivalent width of $\mathrm{H} \beta+[\mathrm{O}$ III] 4959/5007 $\AA$ lines can be well over $1000 \AA$, and could reach as high as 2000-3000 $\AA$ in some cases (e.g., Smit et al. 2014). This suggests that although the NIRCam LW grism mode is not as sensitive as NIRSpec, it may be quite effective in finding high-redshift galaxies through blind search for strong line emitters (note the high signal-to-noise emission lines in Figure 5).

\subsection{Wide-field Slitless Spectroscopy of Dark Clouds}

Sun-like stars form in nearby dark clouds within our galaxy; the gas and dust within these regions is pulled together by gravity until the central regions form stars. These gravitationally-confined dark globules are very dense and emit no visible light, but they can be studied using the infrared light of background stars. The amount of dust (silicate and carbon) and gas material in these clumps can be measured by observing the brightness of these background stars at several different infrared wavelengths using NIRCam filter imaging. Likewise the amount of $\mathrm{H}_{2} \mathrm{O}, \mathrm{CO}$, and $\mathrm{CO}_{2}$ ice on the cloud's dust grains can be measured with NIRCam LW grism spectra, as simulated in Figure 6 for the B335 cloud. Background stars in high extinction regions show little overlap in their relatively short spectra through F430M $\left(\mathrm{CO}_{2}\right)$ or F460M (CO) filters, and most overlaps can be resolved with additional observations taken with the grism having dispersion in the perpendicular direction. Observations like these will show how the density of star-forming clouds changes with their radius and how volatile materials freeze out onto dust grains.

\subsection{Spectra of Transiting Planets}

We have used our 1-D spectral time-series simulator to model the expected JWST spectra of several transiting planets and estimate their scientific information content. ${ }^{9}$ We have recently extended this code to include simulations of SW DHS data, and we show an example of a combined SW DHS and LW grism spectrum in Figure 7. The model transmission spectrum of a hot Jupiter planet is shown as a solid red curve, and the simulated data have been binned to spectroscopic resolution $\mathrm{R}=100$ and are shown as red data points with error bars indicating their uncertainties. These observations would have to be made during at least two separate transits: one transit with a grism plus F322W2 filter, one with a grism and the F444W filter, and SW DHS observations made simultaneously with at least one of the LW grism exposures. Strong absorptions (large 

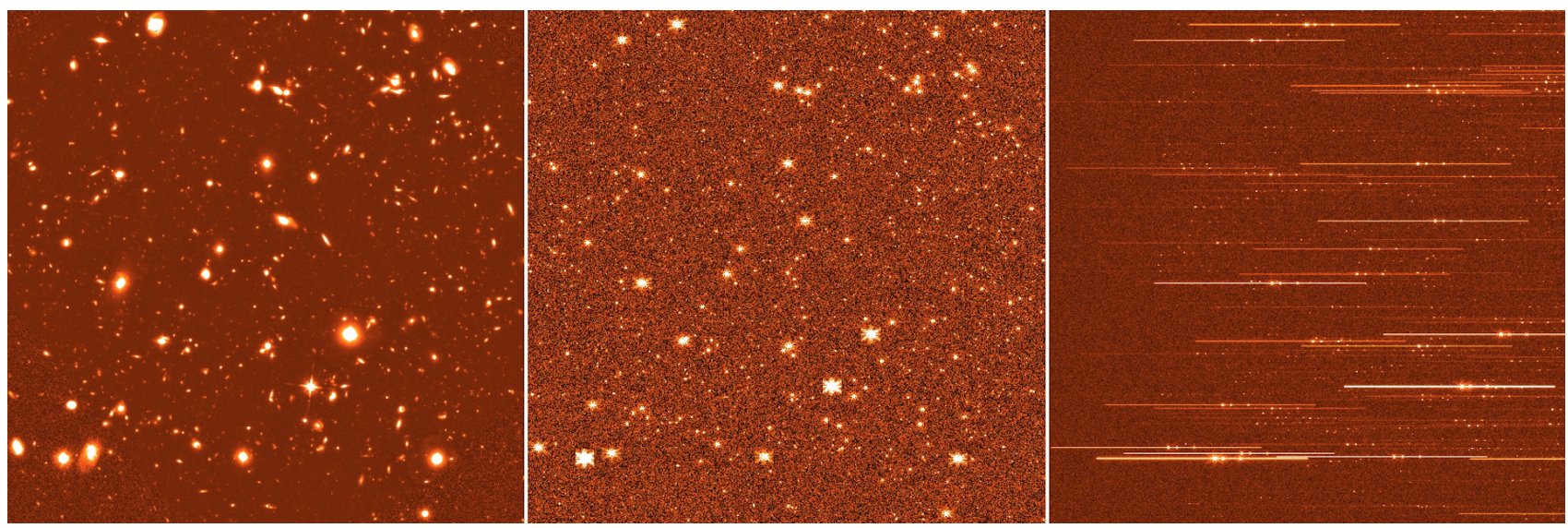

Figure 5. Left: HST/WFC3-IR F160W image of the Hubble eXtreme Deep Field ${ }^{10}$ (XDF) covering the area around the Hubble Ultra-Deep Field (HUDF). The total exposure time is $~ 65$ hours over most of the image; Center: 2-hour JWST NIRcam image in the F356W filter simulated with the source catalog produced from the XDF F160W image on the left. For simplicity, all the sources were assumed to be point-like (i.e., represented by the simulated WebbPSF F356W PSF) and $m_{356, A B}=m_{160, A B}$; Right: Simulated 2-hour NIRCam LW grism data in the F356W filter and R grism. The input model spectrum was constructed as a combination of a flat $f_{\nu}$ continuum and three emission lines, $\mathrm{H} \beta$ and [O III] $4959 / 5007 \AA$ lines, with rest-frame equivalent widths of $\sim 180,200$, and $600 \AA$, respectively (the line widths were assumed to be unresolved). All the sources were placed at $z=6$ to illustrate the detectability of the three emission lines as function of source brightness (wavelength increases toward left).

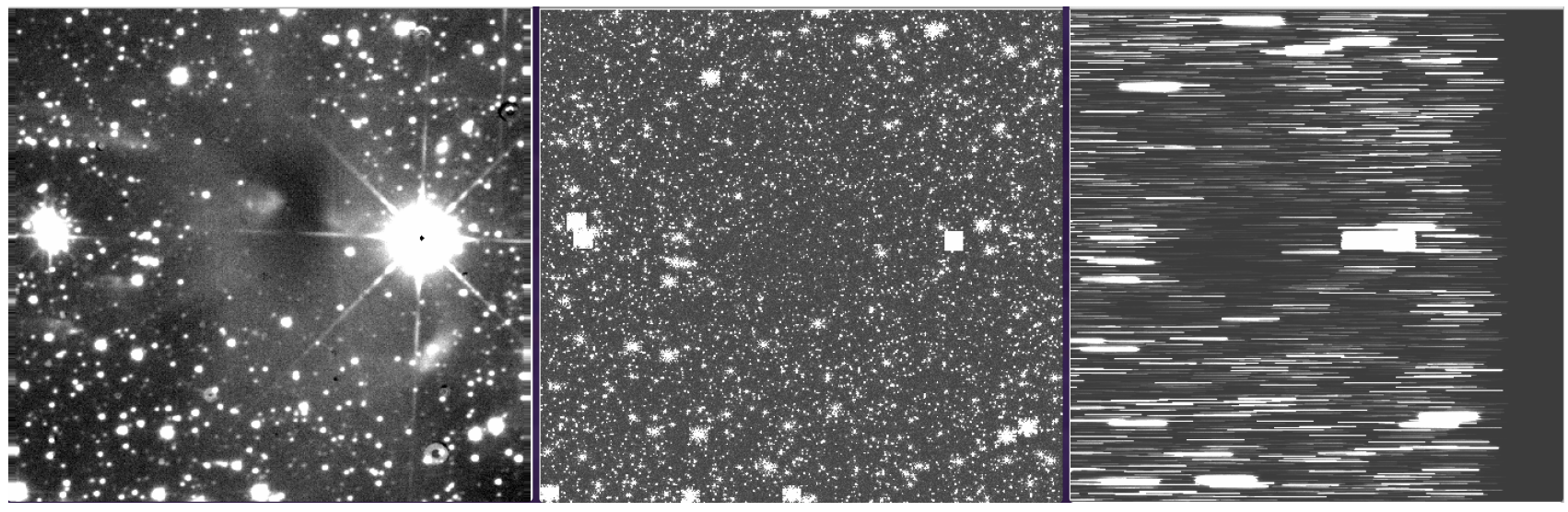

Figure 6. Left: B335 dark coud $K$-band $(\lambda=2.2 \mu \mathrm{m})$ UKIRT image with 3.2 hr integration time. Center: Simulated 1 second JWST NIRCam image in the F430M filter, using simulated WebbPSF PSFs (see http://www.stsci.edu/wfirst/software/webbpsf) for the stars seen in the UKIRT data and also added fainter stars. Right: Simulated 30 second NIRCam F430M R grism image, showing the spectra of the individual stars seen in the images. Background stars in high extinction regions show little overlap in their relatively short spectra, and most overlaps can be resolved with additional observations taken with the $\mathrm{C}$ grism. This spectral region will show $4.3 \mu \mathrm{m} \mathrm{CO}_{2}$ absorptions from ice mantles forming on dust grains in the cloud. 


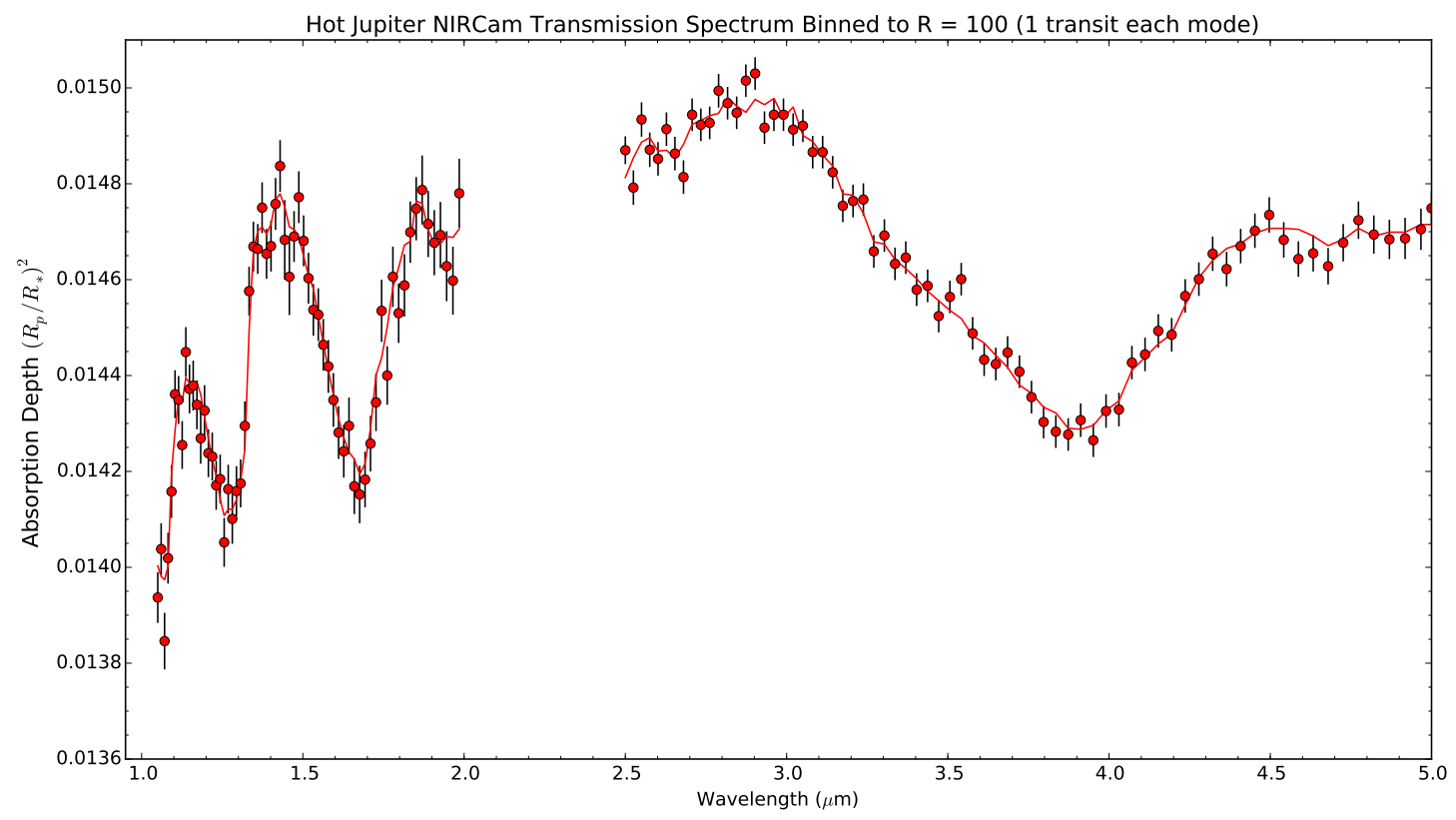

Figure 7. Simulated NIRCam DHS plus LW grism transmission observation of a model hot Jupiter planet with a clear solar composition atmosphere and the system parameters of HD 209458b. The transmission model is shown as the solid red curve, and simulated data are shown as points with error bars denoting uncertainties. A single transit plus equal time on the star alone was simulated at each wavelength, and 2 DHS spectra are co-added, appropriate for a 256 row detector subarray. Covering this spectral range would require observations of at least 2 transits (see Section 4.3). We note that DHS use is not yet supported for scientific observations.

absorption depth values) of $\mathrm{H}_{2} \mathrm{O}$ are clearly seen at $1.2,1.4,1.9$, and $3.0 \mu \mathrm{m}$, with CO at $4.6 \mu \mathrm{m}$. These observations should constrain the $\mathrm{H}_{2} \mathrm{O}$ and $\mathrm{CO}$ mixing fractions of similar transiting planets to factors of $2-3$, much better than HST or other current observations. ${ }^{9}$

\section{OBSERVATION PLANNING}

We intend for the information provided above to be sufficient for assessing the basic feasibility of most NIRCam slitless spectroscopic observations. Planning tools for detailed assessment of sensitivity in specific instrument configurations (as explained in previous sections) and for designing observation sequences will be available through the Space Telescope Science Institute (STScI), the JWST science operations center. Simultaneous SW imaging and LW grism observations will be supported for Cycle 1 observations, but the schedule for implementing simultaneous SW DHS plus LW grism observations is not currently known.

A prototype exposure time calculator (ETC) is currently available for estimating sensitivity and needed exposure times, but it does not support the LW grism mode or imaging through the SW weak lens. A new, scene-based ETC (Pandeia) will support LW grism and all other major JWST observation modes when it is released in January 2017. As described in Section 4, we can also provide NIRCam-specific configuration files for the aXeSIM package that can be used to simulate NIRCam LW grism observations and estimate their signalto-noise. Once the needed integration time has been calculated (e.g., from Pandeia, aXeSIM, or Table 1), observation details can be planned with the Astronomer's Proposal Tool (APT $\left.{ }^{\S}\right)$ for JWST. The current version of APT includes a template for the grism single-object time-series observation mode, including simultaneous imaging in the SW channel. The template for planning wide-field slitless spectroscopy observations is being

\footnotetext{
$\S_{\text {see http://www.stsci.edu/hst/proposing/apt }}$
} 
designed now, and will be available by early 2017. These templates allow selection of targets, detector readout (and subarray) parameters, filters, grisms, and (for the wide-field mode) mosaics and dithers. APT provides estimates of observing windows for targets (consistent with the nominal launch date, orbit, and field of regard restrictions), as well as observing overheads.

\section{ACKNOWLEDGMENTS}

We thank D. Coe for preparing the figure showing the layout of grism spectra in Figure 1 and S. Lilly for discussions and assistance with determining the LW grism dispersion orientations. We also thank M. Line for the exoplanet transmission model and N. Lewis for operations information. TPG and coauthors acknowledge funding support by the NASA JWST project for NIRCam, NASA WBS 411672.05.05.02.02.

\section{REFERENCES}

[1] Beichman, C. A., Rieke, M., Eisenstein, D., Greene, T. P., Krist, J., McCarthy, D., Meyer, M., and Stansberry, J., "Science opportunities with the near-IR camera (NIRCam) on the James Webb Space Telescope (JWST)," in [Space Telescopes and Instrumentation 2012: Optical, Infrared, and Millimeter Wave], Proc. SPIE 8442, 84422N (Sept. 2012).

[2] Krist, J. E., Beichman, C. A., Trauger, J. T., Rieke, M. J., Somerstein, S., Green, J. J., Horner, S. D., Stansberry, J. A., Shi, F., Meyer, M. R., Stapelfeldt, K. R., and Roellig, T. L., "Hunting planets and observing disks with the JWST NIRCam coronagraph," in [Techniques and Instrumentation for Detection of Exoplanets III], Proc. SPIE 6693, 66930H (Sept. 2007).

[3] Rieke, M. J., Kelly, D., and Horner, S., "Overview of James Webb Space Telescope and NIRCam's Role," in [Cryogenic Optical Systems and Instruments XI], Heaney, J. B. and Burriesci, L. G., eds., Proc. SPIE 5904, 1-8 (Aug. 2005).

[4] Greene, T., Beichman, C., Eisenstein, D., Horner, S., Kelly, D., Mao, Y., Meyer, M., Rieke, M., and Shi, F., "Observing exoplanets with the JWST NIRCam grisms," in [Techniques and Instrumentation for Detection of Exoplanets III], Proc. SPIE 6693, 66930G (Sept. 2007).

[5] Jaffe, D. T., Wang, W., Marsh, J. P., Deen, C. P., Kelly, D., and Greene, T. P., "Fabrication and test of silicon grisms for JWST-NIRCam," in [Space Telescopes and Instrumentation 2008: Optical, Infrared, and Millimeter], Proc. SPIE 7010, 70103L (July 2008).

[6] Shi, F., King, B. M., Sigrist, N., and Basinger, S. A., "NIRCam Long Wavelength Channel grisms as the Dispersed Fringe Sensor for JWST segment mirror coarse phasing," in [Space Telescopes and Instrumentation 2008: Optical, Infrared, and Millimeter], Proc. SPIE 7010, 70102E (July 2008).

[7] Erickson, E. F. and Rabanus, D., "Beam shape effects on grating spectrometer resolution," Applied Optics 39, 4486-4489 (May 2000).

[8] Beichman, C., Benneke, B., Knutson, H., Smith, R., Lagage, P.-O., Dressing, C., Latham, D., Lunine, J., Birkmann, S., Ferruit, P., Giardino, G., Kempton, E., Carey, S., Krick, J., Deroo, P. D., Mandell, A., Ressler, M. E., Shporer, A., Swain, M., Vasisht, G., Ricker, G., Bouwman, J., Crossfield, I., Greene, T., Howell, S., Christiansen, J., Ciardi, D., Clampin, M., Greenhouse, M., Sozzetti, A., Goudfrooij, P., Hines, D., Keyes, T., Lee, J., McCullough, P., Robberto, M., Stansberry, J., Valenti, J., Rieke, M., Rieke, G., Fortney, J., Bean, J., Kreidberg, L., Ehrenreich, D., Deming, D., Albert, L., Doyon, R., and Sing, D., "Observations of Transiting Exoplanets with the James Webb Space Telescope (JWST)," Publications of the Astronomical Society of the Pacific 126, 1134-1173 (Dec. 2014).

[9] Greene, T. P., Line, M. R., Montero, C., Fortney, J. J., Lustig-Yaeger, J., and Luther, K., "Characterizing Transiting Exoplanet Atmospheres with JWST," The Astrophysical Journal 817, 17 (Jan. 2016).

[10] Illingworth, G. D., Magee, D., Oesch, P. A., Bouwens, R. J., Labbé, I., Stiavelli, M., van Dokkum, P. G., Franx, M., Trenti, M., Carollo, C. M., and Gonzalez, V., "The HST eXtreme Deep Field (XDF): Combining All ACS and WFC3/IR Data on the HUDF Region into the Deepest Field Ever," The Astrophysical Journal Supplement 209, 6 (Nov. 2013). 
[11] Smit, R., Bouwens, R. J., Labbé, I., Zheng, W., Bradley, L., Donahue, M., Lemze, D., Moustakas, J., Umetsu, K., Zitrin, A., Coe, D., Postman, M., Gonzalez, V., Bartelmann, M., Benítez, N., Broadhurst, T., Ford, H., Grillo, C., Infante, L., Jimenez-Teja, Y., Jouvel, S., Kelson, D. D., Lahav, O., Maoz, D., Medezinski, E., Melchior, P., Meneghetti, M., Merten, J., Molino, A., Moustakas, L. A., Nonino, M., Rosati, P., and Seitz, S., "Evidence for Ubiquitous High-equivalent-width Nebular Emission in z $~ 7$ Galaxies: Toward a Clean Measurement of the Specific Star-formation Rate Using a Sample of Bright, Magnified Galaxies," The Astrophysical Journal 784, 58 (Mar. 2014). 